\title{
Effective normalization for copy number variation detection from whole genome sequencing
}

\author{
Angel Janevski, Vinay Varadan, Sitharthan Kamalakaran, Nilanjana Banerjee, Nevenka Dimitrova* \\ From IEEE International Workshop on Genomic Signal Processing and Statistics (GENSIPS) 2011 \\ San Antonio, TX, USA. 4-6 December 2011
}

\begin{abstract}
Background: Whole genome sequencing enables a high resolution view of the human genome and provides unique insights into genome structure at an unprecedented scale. There have been a number of tools to infer copy number variation in the genome. These tools, while validated, also include a number of parameters that are configurable to genome data being analyzed. These algorithms allow for normalization to account for individual and population-specific effects on individual genome CNV estimates but the impact of these changes on the estimated CNVs is not well characterized. We evaluate in detail the effect of normalization methodologies in two CNV algorithms FREEC and CNV-seq using whole genome sequencing data from 8 individuals spanning four populations.

Methods: We apply FREEC and CNV-seq to a sequencing data set consisting of 8 genomes. We use multiple configurations corresponding to different read-count normalization methodologies in FREEC, and statistically characterize the concordance of the CNV calls between FREEC configurations and the analogous output from CNV-seq. The normalization methodologies evaluated in FREEC are: GC content, mappability and control genome. We further stratify the concordance analysis within genic, non-genic, and a collection of validated variant regions.

Results: The GC content normalization methodology generates the highest number of altered copy number regions. Both mappability and control genome normalization reduce the total number and length of copy number regions. Mappability normalization yields Jaccard indices in the $0.07-0.3$ range, whereas using a control genome normalization yields Jaccard index values around 0.4 with normalization based on GC content. The most critical impact of using mappability as a normalization factor is substantial reduction of deletion CNV calls. The output of another method based on control genome normalization, CNV-seq, resulted in comparable CNV call profiles, and substantial agreement in variable gene and CNV region calls.
\end{abstract}

Conclusions: Choice of read-count normalization methodology has a substantial effect on CNV calls and the use of genomic mappability or an appropriately chosen control genome can optimize the output of CNV analysis.

\section{Background}

Genetic variation in the human genome occurs in many forms ranging from large chromosomal abnormalities to single nucleotide variations, each with varying functional significance. Copy number variation $(\mathrm{CNV})$ is one such genetic variation that can range from a few kilobases to megabases, and involve deletions, duplications, insertions or translocations. Multiple methodologies on a genome-

\footnotetext{
* Correspondence: angel.janevski@philips.com; nevenka.dimitrova@philips. com

Philips Research, 345 Scarborough Rd, Briarcliff Manor, NY 10510, USA
}

wide scale have been used, including RDA, CGH, and more recently: CNV calling using next generation sequencing [1]. The associations between CNVs and phenotypic variation or disease-susceptibility are increasingly being investigated [2], with the most obvious mechanism being gene-dosage caused by variations in the number of copies of a gene or its associated regulatory elements. However, investigations into the biological implications of CNVs in normal and cancer samples have largely been limited by measurement technologies. Multiple studies have confirmed that the amplifications of oncogenes and loss of
Ciomed Central

C 2012 Janevski et al.; licensee BioMed Central Ltd. This is an open access article distributed under the terms of the Creative Commons Attribution License (http://creativecommons.org/licenses/by/2.0), which permits unrestricted use, distribution, and reproduction in any medium, provided the original work is properly cited. 
tumor suppressors are implicated in the development and progression of cancer. Validation of CNV results has been extremely challenging due to natural CNV variations within and across populations [3-5]. This problem is even more exacerbated in cancer, and it is now recognized that cancers have CNV subtype profiles [6]. Establishing frameworks for evaluation of CNV algorithms is very important both for human diversity studies as well as cancer.

In contrast to microarray-based $\mathrm{CNV}$ detection methods, where probes are designed following a carefully developed protocol, whole genome sequencing reads represent a random sampling from a library and could be susceptible to biases, for example GC content and other biophysical and chemical characteristics. In addition, the structure of the human genome with repeat elements and paralogous stretches of sequences make mapping of individual sequencing reads to a reference genome a non-trivial problem.

Several methods and tools have addressed various aspects of determining $\mathrm{CNV}$ information based on sequencing data, each accounting for different forms of normalization: for example, CNVnator [7] uses a meanshift approach with additional refinements (multiplebandwidth partitioning and GC correction), CNV-seq produces relative copy number profiles from paired genomes with one serving as a reference [8], FREEC uses mappability and a control genome [9], and an interesting application of mrFAST and msFAST [3]. Each tool has a unique approach and is successful in addressing some of the CNV detection challenges. Of the available CNV estimation tools, we selected FREEC and CNV-seq as both tools apply a statistical approach to integrate background information about the genome into making CNV calls. Additionally, FREEC allows for various types of normalizations, which we deemed a key element in analyzing genome sequences from multiple populations. The FREEC tool was developed specifically to enable controlfree copy number alteration detection [9]. FREEC requires the user to only provide the ploidy of the genome in order to assign absolute copy number to each predicted CNV, but FREEC can also be run in other modes which allow for the normalization of read counts in the sample genome by inclusion of a control genome or a mappability track. The algorithm first calculates a raw copy number profile by counting the number of reads that map to non-overlapping windows across the entire genome. The second step involves normalizing these raw read counts to account for sequence characteristics of the genome that could influence the number of reads within each window, followed by segmentation and subsequent copy-number estimations.

We apply FREEC on a set of genomes spanning four populations to assess concordance between the outputs from different normalization methods. We also analyze the same genomes with CNV-seq and we compare the output from both tools.

\section{Methods \\ Whole genome sequencing data}

To characterize the landscape of structure variations, we use whole genome sequencing data from three publicly available genomes of Caucasian (CEU, NA12891; CEf, NA12892 [4]), African (YRI1, NA19239 [6]; YRI2 NA 18507 [10]), and Asian (Yh1 [11]; KOR [12]) individuals, as well as two currently unpublished genomes of two individuals (UG1, and UG2) from a population distinct from the six public genomes. All were sequenced using the Illumina GAII sequencer using paired-end and single-end reads differing primarily in the read length the first three sets genomes are based mostly on 36-base reads, whereas the 2 additional genomes are using 115 base reads. All genomes were processed and aligned for this analysis using the Burrows Wheelers Aligner [13] and Samtools [14] to 25x coverage or higher using a total number of aligned bases ranging from 100 to 275 million bases. Identical assembly pipeline was applied to align the genomes to the Hg19 human genome reference. For validation, we use genic regions as defined in RefGene [15], as well as a 8,599 validated CNV segments from Conrad et al. [5] comparing genotyping information from several populations.

\section{Estimating copy number variation with FREEC}

We run FREEC with $3 \mathrm{~Kb}$ window size and other default parameters except those under study (detailed below). We compared the CNV estimates from the output of FREEC, which applies a sliding window strategy with GC content normalization to make absolute copy number predictions. FREEC first maps reads from a given sequencing run to non-overlapping windows spanning the entire reference genome. The raw copy number of a given genomic region is assumed to be proportional to the number of reads that align to the windows spanning that region. The algorithm then normalizes these raw read counts to account for sequence characteristics of the genomic region which influence the number of reads within each window. A segmentation algorithm is applied to the normalized read counts to identify contiguous windows that make up a genomic region with a unique copy-number value. The final step in the algorithm estimates the copy-number value of the segmented genomic regions, thus resulting in a genome-wide copy number profile.

FREEC can be run in three configurations corresponding to different normalizations of read counts within a window [see Additional File 1]. We compare FREEC results from the different configurations with $\mathrm{CNV}$ estimation based on normalization from: (i) GC content, 
(ii) mappability [16] (76-base segment length for UG1 and UG2, and 36-base segment length for the remaining genomes), and (iii) control genome.

Mappability characterizes the degree to which a region of certain length is distinct and hence uniquely mappable to the reference genome. We use FREEC's default parameter which, given mappability information, considers regions for which $85 \%$ of the bases are mappable. We use three different criteria to select a control genome: (i) inpopulation where all genomes use as control the other genome from the same population, (ii) single control genome YRI1 (African, similar coverage profile as most genome), and (iii) single control genome YRI2 (African, high coverage genome, $>50 \mathrm{X}$ ). For in-population controls, the genomes are paired as follows: UG1 and UG2, YRI1 and YRI2, CEU and CEf; and Yh1 and KOR.

\section{Estimating copy number variation with CNV-seq}

CNV-seq [8] uses a fixed length sliding window and normalization of the analyzed (test) genome using a control genome. Differences in read counts mapped between the control and test genomes for a given segment determine the relative copy number change. We use CNV-seq with two modifications of the default parameters for increased stringency: lower p-value for the CNV calls $\left(10^{-4}\right.$ instead of the default $10^{-3}$ ), and higher number of consecutive windows required to call a variation (6 instead of the default 4). We present results using a 3 kilobase window and the trends remain the same with smaller or larger window sizes (data not shown).

We use two sets of seven CNV-seq comparisons, where CNV analysis was done using YRI1 in the first and YRI2 in the second set. These sets are analogous to the FREEC configurations using the African genomes as control, namely (ii) and (iii) in the previous subsection.

\section{Merging CNV calls into comparative CNV calls}

To enable comparative analysis of CNV segments output by multiple tool configurations, we consolidate the outputs of each of the three FREEC configurations and one CNVseq configuration into a set of CNV segments that characterize variations collectively. The steps in the method for merging the $\mathrm{CNV}$ segment are given in Figure 1A, and are also illustrated with an example in Figure 1B. Briefly, a merged list of CNV segments is generated by aligning all detected CNV segments in a set of genomes and extracting all unique segment start and end positions to generate a new, more granular set of $\mathrm{CNV}$ regions. $\mathrm{CNV}$ values are assigned each to these regions for each genome, based on the overlap of the new segments with the original CNV calls. The merging of segments generates an aggregated view of a set of CNVs, and effectively adds a "second dimension" to the genome resulting from a combined set of genomes, represented with a vector containing the original $\mathrm{CNV}$ values.

In the example in Figure $1 \mathrm{~B}$, the $\mathrm{CNV}$ calls from three configurations are broken into five merged segments $\mathrm{MS}_{\mathrm{n}}-\mathrm{MS}_{\mathrm{r}}$ each of which is characterized with a vector of three values. We call these segments comparative $\mathrm{CNV}(\mathrm{cCNV})$ regions. In the example in Figure 1, two $\mathrm{cCNV}$ are formed $\left(\mathrm{MS}_{\mathrm{n}^{-} \mathrm{r}}\right.$ and $\left.\mathrm{MS}_{\mathrm{p}^{-} \mathrm{q}}\right)$ as a result of this step.

The creation of cCNV segments tends to "pad" the segments in one or more CNV estimates as the segments are merged to meet the outer boundaries of overlapping CNV segments. In our current approach, we focus on encompassing all bases in a shared CNV region. In a more conservative approach, one may consider a more restrictive method to prune the ends of cCNV segments and narrow the overlapping segment. In our approach, due to the tendency of FREEC to merge very large adjacent segments into very long segments (several million bases), we introduce a pre-processing step to fragment segments into 10,000-base segments and avoid superficial extension of $\mathrm{cCNV}$ segments into configurations where a small region was originally detected. In our example, this would remedy a case where the length of $S_{c}$ is relatively small, and $S_{d}$ very large. With the fragmentation step, $S_{d}$ is split into $k$ segments: $S_{d 1}, S_{d 2}, \ldots S_{d k}$ and only a subset of these overlapping with $\mathrm{Sc}$ are returned as an overlapping cCNV region for $\mathrm{CNV}_{2}$ and $\mathrm{CNV}_{3}$. The remaining $\mathrm{S}_{\mathrm{di}}$ are separately considered as a variation only in $\mathrm{CNV}_{3}$.

In a subsequent step, all cCNV segments are annotated for their overlap with the gene regions in refSeq and the CNV regions in the [5], referred to as 'Sanger' in the rest of the text. Based on the overlap, each cCNV region is assigned two values between 0 and 1 ranging between no overlap (0), partial overlap (between 0 and 1 ), and full overlap (1).

To obtain the number of bases in genic regions, we use the following formula: Genic $(c)=\sum_{c \in c C N V \text { regions }}$ length $(c) *$ refSeq_overlap $(c)$ where for each $\mathrm{cCNV}$ region $c$, we scale its length given in number of bases with the fraction of the segment overlapping with the gene (refSeq_overlap). Non-genic regions are simply the inverse of genic regions: NonGenic $(c)=\sum_{c \in c \text { CNV regions }}[$ length $(c)-$ Genic $(c)]$. Finally, to obtain the number of bases overlapping Sanger regions, we use: Sanger $(c)=\sum_{c \in c C N V \text { regions }}$ length $(c) *$ Sanger_overlap $(c)$, where Sanger_overlap indicates the fraction of the segment overlapping with Sanger regions. 
Input: CNV calls in the format <chromosome, start, end> from N FREEC configurations

A. Output: cCNV segments in the format <chromosome, start, end, CCNV_vector>; where cCNV_vector of length $\mathrm{N}$ contains $\mathrm{CNV}$ level for the corresponding FREEC configuration

Steps:

1. Concatenate all CNV calls from $\mathrm{N}$ configurations into all_CNV

2. Sort by chromosome, start, end

3. Start an empty output matrix result with $3+\mathrm{N}$ columns, keep a vectors of size $N$ CNV_levels to hold CNV value for configurations

4. Make the start and end of the first segment ccnv_start and ccnv_end update corresponding CNV_levels with the CNV level the first element in cnv_call

5. For each CNV call cnv_call in all_CNV for each chromosome chr do if cnv_call.start >ccnv_end

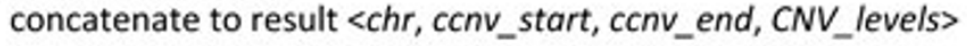
reset $C N V_{-}$levels, update level for $c n v \_c a l l$ ccnv_start and ccnv_end set from cnv_call start and end elseif cnv_call.end $>$ ccnv_end ccnv_end $=$ cnv_call.end update CNV_levels level for cnv_call

B.

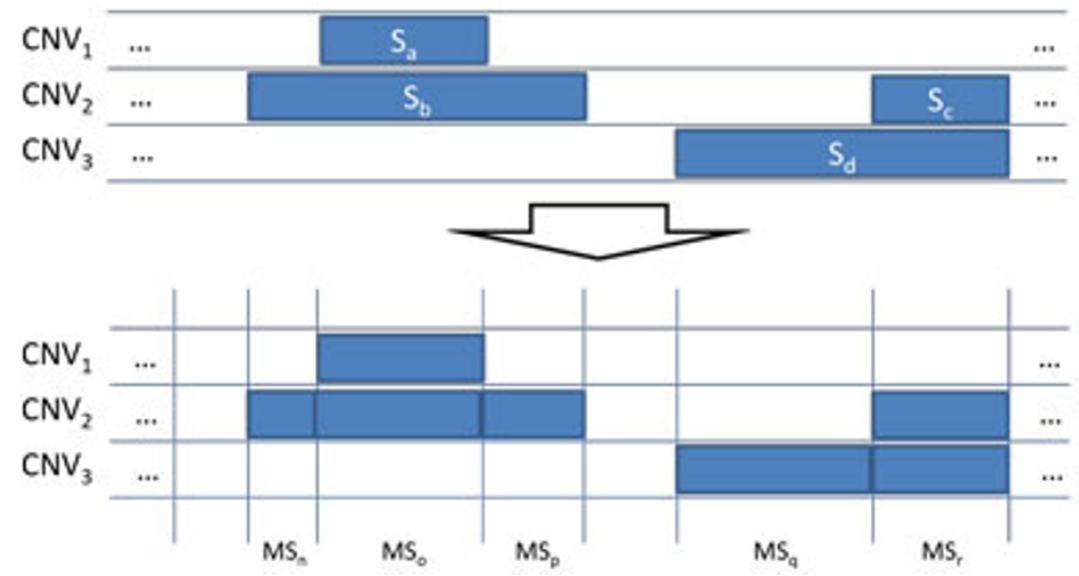

Figure 1 cCNV: Merging of CNV segments from multiple FREEC configurations. A. CCNV algorithm. B. Example of merging CNV calls.

\section{Comparison of configurations and estimating} concordance

Figure 2 depicts our analysis flow which compares the results from the execution of FREEC and CNV-seq. After Alignment, the CNV step applies a selected
FREEC or CNV-seq configuration resulting in CNV calls. The cCNV step merges all the output segments from the configurations. cCNV segments are characterized in the Overlap step to quantify the intersection with genic regions, and 8,599 validated segments from

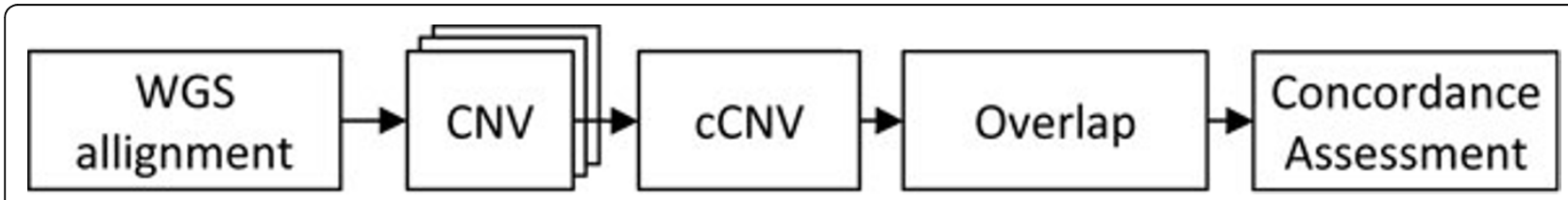

Figure 2 Comparison of analysis outputs. Our analysis pipeline which compares the results from analyzing results from three FREEC modes. cCNV merges all the output regions for the three. CCNV segments are characterized with respect to coverage and overlap overall, in genic and non-genic regions, and in the context of the 8,599 validated segments from [11]. 
the Sanger set. In the last step, Concordance Assessment, we assess concordance between the three FREEC normalizations. We measure the similarity using the Jaccard index (JI) on any two sets of output comparing the sets at the level of individual base pairs. Given two configurations $\mathrm{A}$ and $\mathrm{B}$, we use the following to compute Jaccard Index: $J I\left(\operatorname{conf}_{A}, \operatorname{conf}_{B}\right)=\frac{\left|\operatorname{conf}_{A} \cap \operatorname{conf}_{B}\right|}{\left|\operatorname{conf}_{A} \cup \operatorname{conf} f_{B}\right|}$. It computes the ratio of the total number of $\mathrm{CCNV}$ bases in the intersection of configurations $\mathrm{A}$ and $\mathrm{B}$ over total number of bases in the union of the of the two sets JI is reported for each genome and four CNV segment sets: all regions, genic, non-genic, and Sanger.

\section{Variation profiles of $\mathrm{CCNV}$}

Additionally, for the output of FREEC, the number of bases falling under a range of copy number amplifications and deletions is reported for individual genomes and within segment sets. The output of this characterization is binned using the following breakpoints: less than 2 copies, 2 to 6 copies, and more than 6 copies.

\section{Results}

\section{cCNV regions}

We focus only on the autosomal CNV segments (chromosomes 1 through 22). We transform the $\mathrm{CNV}$ regions called by FREEC to cCNVs. We interchangeably refer to $\mathrm{CNV}$ and $\mathrm{cCNV}$ regions in the remainder of the text all discussion refers to the CNV obtained as a result of combining $\mathrm{CNV}$ regions from FREEC configurations, i.e. one should consider all CNV references to be cCNV. Table 1 provides a summary of the output from three separate control genome choices for FREEC and Table 2 provides a summary for the output of CNV-seq. In FREEC, GC content normalization outputs CNV regions ranging 57-98 $\mathrm{Mb}$ among the eight genomes. Adding mappability as a normalization parameter results in a substantially reduced total number of bases called in comparison to GC content normalization - calling only $25 \%$ of the total base pairs outputted by GC normalization but going as low as 6-7\% in three genomes (CEU, $\mathrm{YH} 1$, and KOR). Adding an in-population control genome as normalization parameter in FREEC yields a smaller reduction of the total lengths ranging from 25 to $60 \%$ with one exception (YRI2, with an over 2-fold increase in total lengths). With YRI1 as a control genome, the total length of base pairs in the control configuration is in the $60 \%$ range for most genomes, with Yh1 at $82 \%$, and CEf at $95 \%$, of the GC content normalization total length, except for an over 2-fold increase with YRI2. Using YRI2 as a control results in a number of variant base pairs comparable to the GC content normalization total length for most genomes, and around $70 \%$ of the total length in YRI1 and CEf. Using YRI1 or YRI2 as a control genome yields to similar summaries in case of mappability and GC content normalization. We observe $\sim 10 \%$ variation due to the "padding" effect discussed earlier.

In $\mathrm{CNV}$-seq, the total length of $\mathrm{CNV}$ regions ranging 22-63 Mb with YRI1 as a control genome and 43-113 $\mathrm{Mb}$ with YRI2 as a control genome. These values are comparable to the ranges in the analogous FREEC runs with control genome normalization.

We then classify the called segments as genic, nongenic and also compare them to a list of commonly occurring variant regions previously reported [3]. Table 3 shows the representation of genic segments in the $\mathrm{CCNV}$ regions. In FREEC as shown in Table 4, genic regions on average constitute $49.7 \%(\mathrm{sd}=17.5 \%)$ in mappability, $17.5 \%(\mathrm{sd}=5.8 \%)$ in GC content, and $27.9 \%(\mathrm{sd}=15.7 \%)$ in control genome normalization. As we change the control genome to YRI1 or YRI2, similar distribution for genic regions is observed for mappability normalization. However, in control normalization, the impact varies: more genic regions are returned in UG1, CEU, and Yh1, but mappability still has more enrichment in genic regions for all genomes.

Table 5 shows the representation of Sanger regions in the total cCNV regions. In FREEC as shown in Table 6, Sanger regions on average account for $14.9 \%$ (sd $=$ $4.9 \%)$ in mappability, $23 \%(\mathrm{sd}=5.7 \%)$ in GC content, and $22.4 \%(\mathrm{sd}=6.8 \%)$ in control genome normalization. Using YRI1 or YRI2 as a control genome, again similar trends are observed for mappability normalization. In control normalization, YRI1 as control increases the representation of these regions in UG1. Using YRI2 as a control substantially reduces the representation of these regions for KOR.

Table 1

\begin{tabular}{|c|c|c|c|c|c|c|c|c|}
\hline $\begin{array}{c}\text { FREEC } \\
\text { Normalization }\end{array}$ & UG1 & UG2 & YRI1 & YRI2 & CEU & CEf & Yh1 & KOR \\
\hline GC content & $77,347,858$ & $79,636,676$ & $97,902,881$ & $70,686,285$ & $76,746,044$ & $89,623,788$ & $78,456,632$ & $57,134,301$ \\
\hline Mappability & $19,520,720$ & $17,252,109$ & $24,758,352$ & $17,768,115$ & $4,925,758$ & $20,064,719$ & $5,697,806$ & $3,931,768$ \\
\hline Control genome (in Population) & $37,100,227$ & $20,683,564$ & $67,291,545$ & $163,831,511$ & $48,113,551$ & $32,010,850$ & $50,189,736$ & $33,322,468$ \\
\hline Control genome (YRI1) & $41,084,695$ & $37,640,839$ & - & $163,831,511$ & $52,945,367$ & $87,318,862$ & $50,727,044$ & $38,000,863$ \\
\hline Control genome (YRI2) & $117,640,623$ & $74,253,250$ & $67,291,545$ & - & $58,461,174$ & $62,120,940$ & $69,839,893$ & $78,225,240$ \\
\hline
\end{tabular}


Table 2

\begin{tabular}{|c|c|c|c|c|c|c|c|c|}
\hline $\begin{array}{c}\text { CNV-seq } \\
\text { Normalization }\end{array}$ & UG1 & UG2 & YRI1 & YRI2 & CEU & CEf & Yh1 & KOR \\
\hline Control genome (YRI1) & $43,235,491$ & $42,881,033$ & - & $63,065,487$ & $22,266,758$ & $27,151,018$ & $25,818,765$ & $38,632,528$ \\
\hline Control genome (YRI2) & $113,662,351$ & $106,834,661$ & $108,082,096$ & - & $59,313,018$ & $49,010,115$ & $79,994,365$ & $43,093,200$ \\
\hline
\end{tabular}

\section{Effect of using mappability track on copy number estimation}

Using mappability tracks significantly alters the number of copy number variants reported. We calculate the Jaccard Index as a measure of similarity between the reported CNVs by FREEC using mappability normalization over GC content normalization. Averaged over the eight genomes, the JI was 0.123 ( $\mathrm{sd}=0.057$ ), indicating low levels of commonality between the reported CNVs (Figure 3A). We annotate the reported calls as genic, non-genic and investigate any differences. Interestingly, $\mathrm{JI}$ is higher in genic regions $($ mean $=0.29, \mathrm{sd}=0.135$ ) than in non-genic regions (mean $=0.074$, sd $=0.039$ ), with a significant difference ( $\mathrm{p}$-value $=0.0006$, Wilcoxon rank sum test). Next, we investigate whether the amplitude of copy number calls varies when mappability is used in normalization (Figure 3B, individual cCNV data is shown in Supplementary Figure 3 [see Additional File 2]). We find significant differences between the reported calls when mappability is used. Using mappability causes a fraction change in the number of CNV deletions reported by FREEC and shift towards higher copy number calls. On average, $70 \%$ of the deletions disappear consistently across the 8 individual genomes ( $\mathrm{sd}=12.5 \%)$. The higher copy number in the range of 2 to 6 copies increases by $108 \%$ in a highly variable fashion ( $\mathrm{sd}=67 \%$ ). Most of these regions appear to be from non-genic areas of the genome.

\section{Effect of using a control genome on copy number estimation}

We observe higher similarity between the copy number variants reported when a control genome is used for normalization as opposed to the mappability criterion shown above. The JI between normalization using a control genome and GC content normalization is $0.384(\mathrm{sd}=0.176)$ when averaged over 8 genomes, with similar values within genic and non-genic annotations of the genome (Figure 4A, also Supplementary Figure 4B and 4C [see

Table 3

\begin{tabular}{ccccccccc}
\hline $\begin{array}{c}\text { CNV-seq } \\
\text { Normalization }\end{array}$ & UG1 & UG2 & YRI1 & YRI2 & CEU & CEf & Yh1 & KOR \\
\hline $\begin{array}{c}\text { Control genome } \\
(\text { YRI1) }\end{array}$ & $46 \%$ & $46 \%$ & - & $55 \%$ & $57 \%$ & $53 \%$ & $61 \%$ & $56 \%$ \\
$\begin{array}{c}\text { Control genome } \\
(\text { YRI2) }\end{array}$ & $55 \%$ & $55 \%$ & $59 \%$ & - & $56 \%$ & $56 \%$ & $54 \%$ & $48 \%$ \\
\hline
\end{tabular}

Additional File 2]). There is no consistent change in the numbers of called deletions or high amplifications between using a control genome and GC content. We observe 0-mean for the fractional change for all regions, with high variability $(\mathrm{sd}=82.2 \%)$. The fraction changes given in Figure 4B (see also, Supplementary Figure 3 [see Additional File 2]) show the copy number range from 2 to 6 exhibiting high fractional change (mean $=85.3 \%$ ) and great variability ( $\mathrm{sd}=102.1 \%)$. This change is most notable in the non-genic regions (mean $=120.2 \%$ and $\mathrm{sd}=139.4 \%$ ). Even more striking is variability in the fractional change of the high copy number in the genic regions $($ mean $=36.6 \%$, sd $=127.8 \%$ ).

In $\mathrm{CNV}$-seq, genic regions are represented in levels comparable to the levels of FREEC with mappability: $53.3 \%(\mathrm{SD}=5.2 \%)$ for YRI1 as a control, and $54.7 \%$ ( $\mathrm{sd}=3.25 \%)$ for YRI2 as a control. Furthermore, we observe higher representation of Sanger regions: $35 \%$ $(\mathrm{sd}=5.4 \%)$ with YRI1 as control, and $27.2 \%(\mathrm{sd}=3.6 \%)$ with YRI2 as control).

\section{Discussion}

The findings presented here have established that a calculation of CNV in whole genome sequencing data without proper normalization with a control genome can be misleading. Much attention needs to be paid to the type of sample, context of the analysis and the population represented in the genome as they may have profound effects on the number of CNV calls. Major challenges in the CNV calling come from the technical difficulty to map regions of the genome with lower mappability and account for repeat regions in a balanced manner. We show that FREEC applied with mappability normalization results in lower concordance between the reported CNVs when compared to GC based normalization. There is

Table 4

\begin{tabular}{ccccccccc}
\hline $\begin{array}{c}\text { FREEC } \\
\text { Normalization }\end{array}$ & UG1 & UG2 & YRI1 & YRI2 & CEU & CEf & Yh1 & KOR \\
\hline $\begin{array}{c}\text { GC content } \\
\text { Mappability }\end{array}$ & $13 \%$ & $12 \%$ & $28 \%$ & $20 \%$ & $12 \%$ & $24 \%$ & $11 \%$ & $21 \%$ \\
$\begin{array}{c}\text { Control genome (in } \\
\text { Population) }\end{array}$ & $10 \%$ & $21 \%$ & $37 \%$ & $51 \%$ & $14 \%$ & $56 \%$ & $13 \%$ & $21 \%$ \\
$\begin{array}{c}\text { Control genome } \\
(\text { YRI1) }\end{array}$ & $22 \%$ & $21 \%$ & - & $51 \%$ & $21 \%$ & $38 \%$ & $17 \%$ & $25 \%$ \\
$\begin{array}{c}\text { Control genome } \\
(Y R I 2)\end{array}$ & $26 \%$ & $24 \%$ & $37 \%$ & - & $17 \%$ & $32 \%$ & $22 \%$ & $23 \%$ \\
\hline
\end{tabular}


Table 5

\begin{tabular}{ccccccccc}
\hline $\begin{array}{c}\text { CNV-seq } \\
\text { Normalization }\end{array}$ & UG1 & UG2 & YRI1 & YRI2 & CEU & CEf & Yh1 & KOR \\
\hline $\begin{array}{c}\text { Control genome } \\
\text { (YRI1) }\end{array}$ & $34 \%$ & $34 \%$ & - & $29 \%$ & $41 \%$ & $43 \%$ & $37 \%$ & $27 \%$ \\
$\begin{array}{c}\text { Control genome } \\
(\text { YRI2) }\end{array}$ & $24 \%$ & $24 \%$ & $24 \%$ & - & $28 \%$ & $34 \%$ & $26 \%$ & $31 \%$ \\
\hline
\end{tabular}

enrichment of CNVs in genic regions - in most individuals genic CNVs encompass about $30-50 \%$ of the total CNVs (and they represent about 1\% of the genome). Furthermore, in mappability normalization, there is significantly higher concordance in the genic regions which could be due to the fact that the mappability of genic regions is typically higher. The coarse definition of mappability in FREEC considers equally all bases with nonzero mappability score is better suited for regions that exhibit higher mappability. Therefore, with non-genic regions likely to have lower mappability scores, this criterion filters out fewer non-genic regions that are overall with lower mappability.

We observe that GC content based normalization in FREEC is likely not accounting for the expected lower number of reads within regions of lower mappability. Algorithms that attempt to call absolute copy number are susceptible to deletion-bias since they typically compare read-counts in specific regions against the background read-count distribution across the whole genome within a single sequencing run. Algorithms implicitly assume that reads are generated uniformly from across the whole genome with read-counts within any given window therefore following a Poisson distribution [4]. However, it is essential to note that while reads are likely generated uniformly from across the genome, they are not necessarily mapped uniformly across the genome even in a purely diploid sample. Regions of the genome with lower mappability tend to accumulate lower read-counts, thus leading to read-count artifacts that can be interpreted as deletions in the absence of proper read-count normalization techniques. Algorithms that attempt to call relative copy numbers, such as CNV-seq, are less prone to mappability artifacts although they suffer from run-specific sources of

Table 6

\begin{tabular}{cccccccccc}
\hline $\begin{array}{c}\text { FREEC } \\
\text { Normalization }\end{array}$ & UG1 & UG2 & YRI1 & YRI2 & CEU & CEf & Yh1 & KOR \\
\hline GC content & $19 \%$ & $21 \%$ & $18 \%$ & $25 \%$ & $21 \%$ & $19 \%$ & $23 \%$ & $38 \%$ \\
$\quad$ Mappability & $19 \%$ & $19 \%$ & $5 \%$ & $16 \%$ & $12 \%$ & $9 \%$ & $18 \%$ & $21 \%$ \\
$\begin{array}{c}\text { Control genome } \\
\text { (in Population) } \\
\begin{array}{c}\text { Control genome } \\
\text { (YRI1) }\end{array}\end{array}$ & $15 \%$ & $28 \%$ & $20 \%$ & $12 \%$ & $22 \%$ & $21 \%$ & $23 \%$ & $37 \%$ \\
$\begin{array}{c}\text { Control genome } \\
(\text { YRI2) }\end{array}$ & $15 \%$ & $22 \%$ & $20 \%$ & - & $25 \%$ & $19 \%$ & $24 \%$ & $26 \%$ \\
\hline
\end{tabular}

read-count variability. Indeed, 99\% of the output from CNV-seq is regions that FREEC considers highlymappable.

The use of a control genome to normalize the readcounts could be thought of as a possible surrogate for a mappability track since presumably regions of poor mappability wouldn't change from the sample genome to the control genome. Furthermore, it could be hypothesized that the control genome ought to be chosen from within the same population as the sample genome. For the FREEC output, we estimated the Jaccard similarity indices of the control-genome based $\mathrm{CNV}$ calls with the GC content based CNV calls for each of the 8 samples by using an in-population control genome and compared these with the respective values of the mappability based CNV calls (Figure 5A and Supplementary Figure 5 [see Additional File 2]). We observe increased variation in the JI values for in-population control genome based normalization when compared to the mappability based normalization. Replacing the inpopulation control genome with a single control genome for all samples, such as the use of YRI1 as a universal control, results in relatively smaller variation in JI values (Figure 5B). Furthermore, choosing a universal control with much higher average coverage of the genome than the sample yields higher similarity of $\mathrm{CNV}$ profiles to the GC content normalization (Figure $5 \mathrm{C}$ ). In other words, if the aim is to maintain higher agreement with the CNV calls out of the GC content normalization procedure, a control genome with similar average coverage as the sample genome (YRI1) might be preferable to a control genome with much higher average coverage (YRI2). Further investigation is needed to determine whether the choice of control genomes affects false positive rates. Relative concordance is worst when we look at the genic regions (Supplementary Figure 5 [see Additional File 2]) and in-population control, both mappability and population-specific variations contributing to this effect. Mappability restricts the concordance most tightly in the Sanger regions - possibly due to the fact that these regions have been already mapped and validated in many individuals - so the individual and population variability affects these results the least.

Finally, when the output of FREEC is compared to the output of $\mathrm{CNV}$-seq, we observe that an explicit normalization (a control genome), also introduces an implicit mappability normalization to the extent of FREEC's use of mappability. The level of agreement in terms of called variant genes and Sanger CNVs between FREEC and CNV-seq are given in Supplementary Tables 1 and 2 [see Additional File 2]. While we observe comparable CNV call profiles between effectively normalized FREEC and $\mathrm{CNV}$-seq, typically CNV-seq would call in the order of $40-50 \%$ of the genes or regions called by FREEC. 


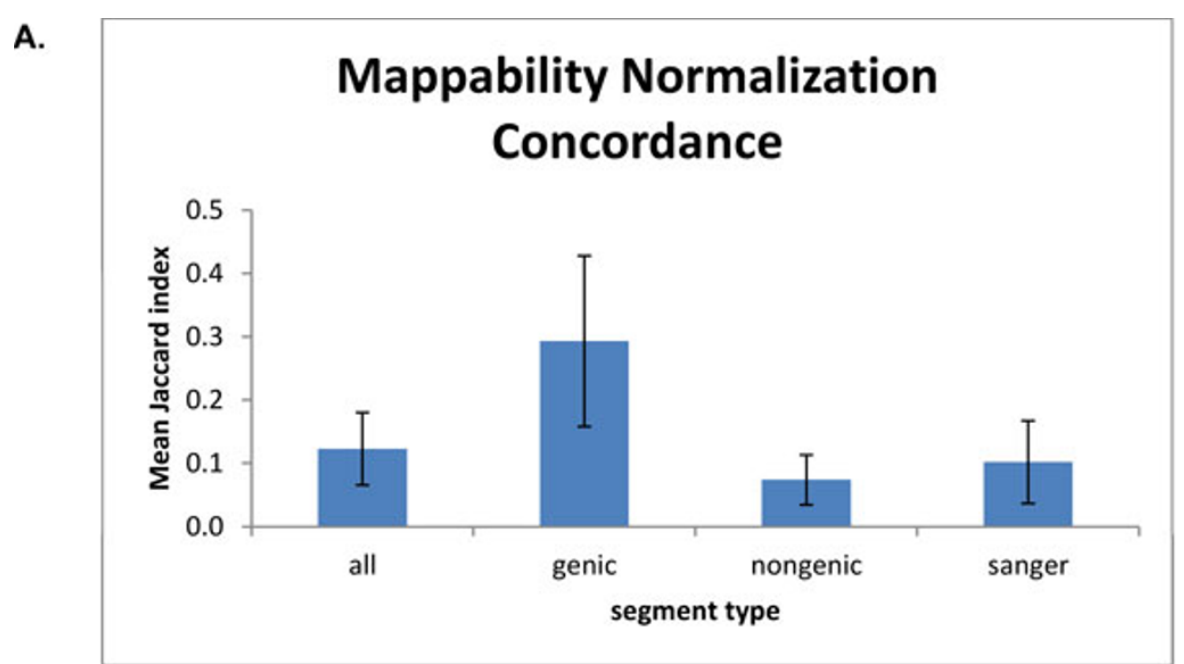

\section{B.}
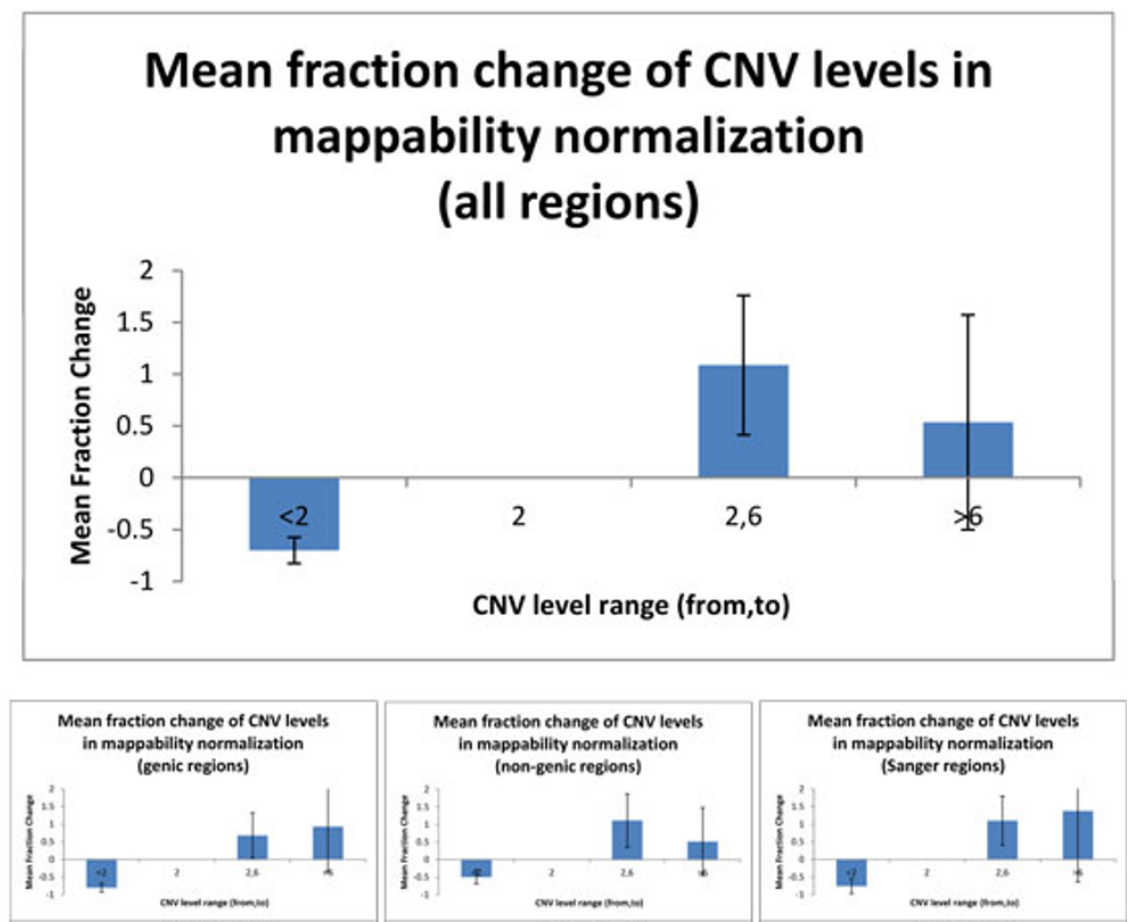

Figure 3 Summary of mappability concordance. A. Summary of mappability concordance for the eight genomes given separately for all, genic, non-genic, and Sanger regions. cCNVs are computed using in-population control. B. Mean fraction change with mappability normalization at three levels: below 2 copies, 2-6 copies and more than 6 copies.

\section{Conclusions}

We observe the effects of adding mappability and control genomes for normalization and we find that both types of normalization improve on the CNVs called by FREEC, with similar CNV call profiles observed in CNV-seq. Extending other tools with similar capability to normalize the analyzed sequence would likely yield similar improvement in concordance. The improvement in concordance is evident with control genome normalization in FREEC and to some extent with mappability normalization. We have some reservation with FREEC's current mappability normalization, as it does not filter out all regions with lower mappability scores - a more elaborate criterion for the mappability filter (e.g. average mappability score) we believe should yield performance comparable to control normalization. Control genome normalization typically introduces more new information to the CNV calls especially in the higher copy number regions. This effect is 
A.

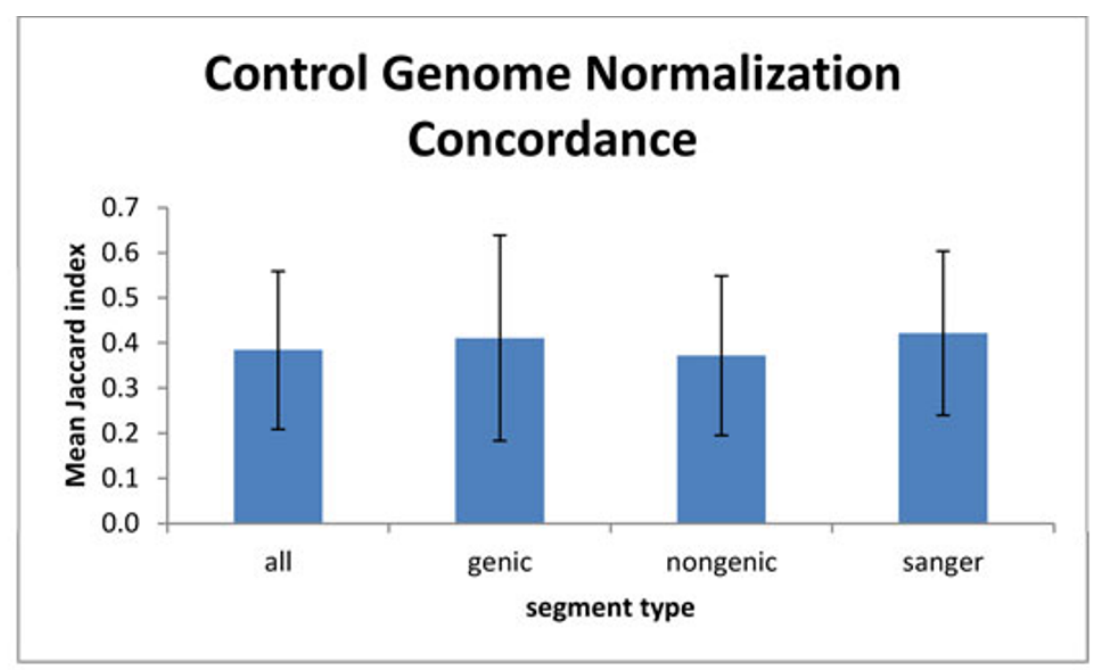

B.
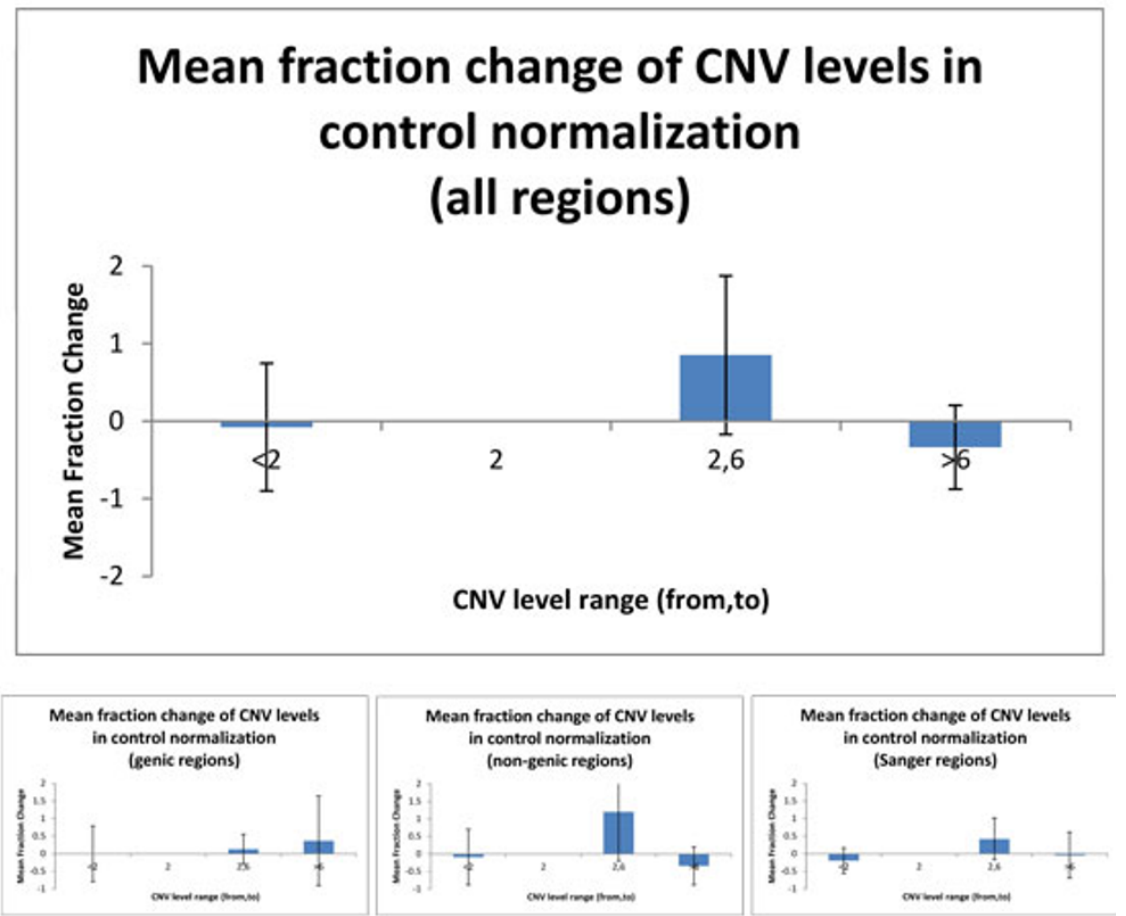

Figure 4 Summary of control genome concordance. A. Summary of control concordance for the eight genomes given separately for all, genic, non-genic, and Sanger regions. cCNVs are computed using in-population control.B. Mean fraction change with control normalization at three levels: below 2 copies, 2-6 copies and more than 6 copies.

smaller in genic regions and in majority of the genomes when considering the validated Sanger segments.

The implication of our analysis is that CNV estimation algorithms that use sequencing data should extend their methods to account for mappability and other sources of variation in the context of multiple sequences and integrate this input into CNV call assessments. Notably, steps should be taken to normalize the output in the context of the population of origin as studies show population-specific variations that may not be accounted for by generic normalization approaches.

Understanding and optimizing CNV methods and tools will be essential as whole genome sequencing will be entering clinical practice in the near future with the promise of characterizing tumor samples down to a single cell. Robust and repeatable methods will be essential 


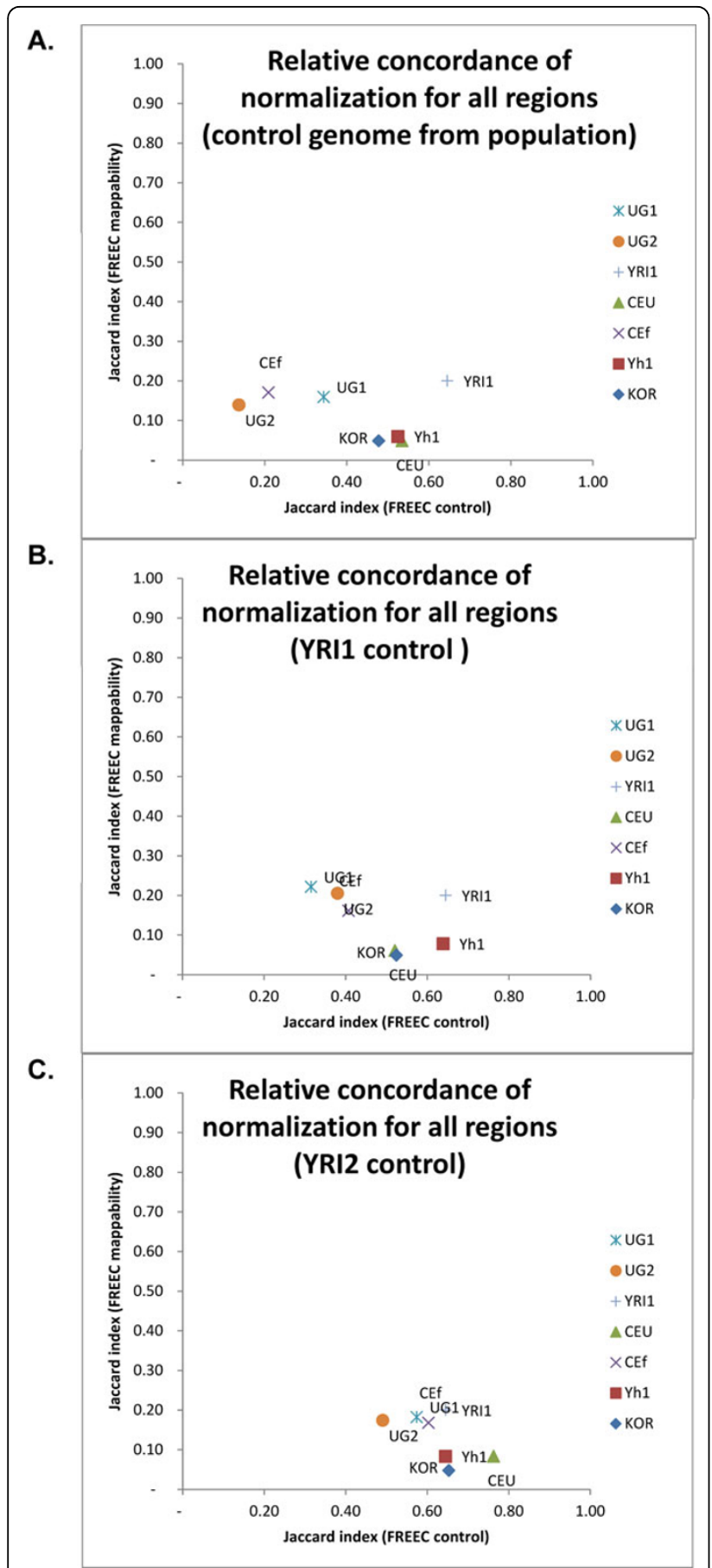

Figure 5 Relative concordance for all CNV regions. A. Jaccard indices of control vs. mappability normalization - control genome from population. B. Jaccard indices of control vs. mappability normalization - control genome isYRI1C. Jaccard indices of control vs. mappability normalization -control genome is YRI2.

for accurate tracking of the progression of cancer and also characterization in the context of other sampled tumors.

\section{Additional material}

Additional file 1: FREEC normalization options. Normalization in FREEC.

Additional file 2: Supplementary figures and tables. Additional

figures and tables referenced in the main document.

\section{Acknowledgements}

Based on "Comparative copy number variation from whole genome sequencing", by Angel Janevski, Vinay Varadan, Sitharthan Kamalakaran, Nilanjana Banerjee and Nevenka Dimitrova which appeared in Genomic Signal Processing and Statistics (GENSIPS), 2011 IEEE International Workshop on. (c) 2011 IEEE [17].

This article has been published as part of BMC Genomics Volume 13 Supplement 6, 2012: Selected articles from the IEEE International Workshop on Genomic Signal Processing and Statistics (GENSIPS) 2011. The full contents of the supplement are available online at http://www. biomedcentral.com/bmcgenomics/supplements/13/S6.

\section{Authors' contributions}

AJ implemented and executed FREEC and CCNV generation and annotation; ND/AJ executed the whole genome assembly and coverage computation. All authors contributed in the analysis and interpretation of the result and writing the text.

\section{Competing interests}

The authors declare that they have no competing interests.

Published: 26 October 2012

\section{References}

1. Alkan C, Coe BP, Eichler EE: Genome structural variation discovery and genotyping. Nat Rev Genet 2011, 12(5):363-76

2. Stankiewicz P, Lupski JR: Structural variation in the human genome and its role in disease. Annu Rev Med 2010, 61:437-455.

3. Sudmant PH, Kitzman JO, Antonacci F, Alkan C, Malig M, Tsalenko A, Sampas N, Bruhn L, Shendure J, 1000 Genomes Project Eichler EE: Diversity of Human Copy Number Variation and Multicopy Genes. Science 2010, 330(6004):641-6.

4. The 1000 Genomes Project Consortium: A map of human genome variation from population-scale sequencing. Nature 2010, 467:1061-1073.

5. Conrad DF, Pinto D, Redon R, Feuk L, Gokcumen O, Zhang Y, Aerts J, Andrews TD, Barnes C, Campbell P, Fitzgerald T, Hu M, Ihm CH, Kristiansson K, Macarthur DG, Macdonald JR, Onyiah I, Pang AW, Robson S, Stirrups K, Valsesia A, Walter K, Wei J, Wellcome Trust Case Control Consortium, Tyler-Smith C, Carter NP, Lee C, Scherer SW, Hurles ME: Origins and functional impact of copy number variation in the human genome. Nature 2010, 464:704-712.

6. Russnes HG, Navin N, Hicks J, Borresen-Dale AL: Insight into the heterogeneity of breast cancer through next-generation sequencing. J Clin Invest 2011, 121(10):3810-8.

7. Abyzov A, Urban AE, Snyder M, Gerstein M: CNVnator: An Approach to Discover, Genotype and Characterize Typical and Atypical CNVs from Family and Population Genome Sequencing. Genome Res 2011, 21:974-984.

8. Xie C, Tammi MT: CNV-seq, a new method to detect copy number variation using high-throughput sequencing. BMC Bioinformatics 2009, 10:80.

9. Boeva V, Popova T, Bleakley K, Chiche P, Cappo J, Schleiermacher G, Janoueix-Lerosey I, Delattre O, Barillot E: Control-free calling of copy number alterations in deep-sequencing data using GC-content normalization. Bioinformatics 2011, 27(2):268-9.

10. Bentley DR, Balasubramanian S, Swerdlow HP, Smith GP, Milton J, Brown CG, Hall KP, Evers DJ, Barnes CL, Bignell HR, Boutell JM, Bryant J, Carter RJ, Keira Cheetham R, Cox AJ, Ellis DJ, Flatbush MR, Gormley NA, Humphray SJ, Irving LJ, Karbelashvili MS, Kirk SM, Li H, Liu X, Maisinger KS, Murray LJ, Obradovic B, Ost T, Parkinson ML, Pratt MR, et al: Accurate 
whole human genome sequencing using reversible terminator chemistry. Nature 2008, 456(7218):53-9.

11. Wang J, Wang W, Li R, Li Y, Tian G, Goodman L, Fan W, Zhang J, Li J, Zhang J, Guo Y, Feng B, Li H, Lu Y, Fang X, Liang H, Du Z, Li D, Zhao Y, Hu Y, Yang Z, Zheng H, Hellmann I, Inouye M, Pool J, Yi X, Zhao J, Duan J, Zhou Y, Qin J, Ma L, et al: The diploid genome sequence of an Asian individual. Nature 2008, 456:60-65.

12. Ahn SM, Kim TH, Lee S, Kim D, Ghang H, Kim DS, Kim BC, Kim SY, Kim WY, Kim C, Park D, Lee YS, Kim S, Reja R, Jho S, Kim CG, Cha JY, Kim KH, Lee B, Bhak J, Kim SJ: The first Korean genome sequence and analysis: Full genome sequencing for a socio-ethnic group. Genome Research 2009, 19(9):1622-9.

13. Li H, Durbin R: Fast and accurate short read alignment with BurrowsWheeler Transform. Bioinformatics 2009, 25:1754-60.

14. Li H, Handsaker B, Wysoker A, Fennell T, Ruan J, Homer N, Marth G, Abecasis G, Durbin R, 1000 Genome Project Data Processing Subgroup: The Sequence alignment/map (SAM) format and SAMtools. Bioinformatics 2009, 25:2078-9.

15. National Library of Medicine (US), National Center for Biotechnology Information: The NCBI handbook [Internet], 2002, Chapter 18, The Reference Sequence (RefSeq) Project.

16. The GEM library. [http://sourceforge.net/apps/mediawiki/gemlibrary].

17. Janevski A, Varadan V, Kamalakaran S, Banerjee N, Dimitrova N: Comparative copy number variation from whole genome sequencing. Genomic Signal Processing and Statistics (GENSIPS), 2011 IEEE International Workshop on: 4-6 December 2011 2011, 123-126.

\section{doi:10.1186/1471-2164-13-S6-S16}

Cite this article as: Janevski et al.: Effective normalization for copy number variation detection from whole genome sequencing. $B M C$ Genomics 2012 13(Suppl 6):S16.

\section{Submit your next manuscript to BioMed Central and take full advantage of:}

- Convenient online submission

- Thorough peer review

- No space constraints or color figure charges

- Immediate publication on acceptance

- Inclusion in PubMed, CAS, Scopus and Google Scholar

- Research which is freely available for redistribution

Submit your manuscript at www.biomedcentral.com/submit 